\title{
Improvement of Lipid Metabolism and Ovalbumin-Induced Type I Allergy by Use of Soybean Milk Fermented by 16 Indigenous Lactic Acid Bacteria
}

\author{
Yuichi Nodake*, Ryoko Miura, Hirokazu Ryoya, Rina Momii, Satomi Toda, Ryuzo Sakakibara \\ Department of Biochemistry, Faculty of Pharmaceutical Sciences, Nagasaki International University, Sasebo, Nagasaki, Japan \\ Email address: \\ nodake@niu.ac.jp (Y. Nodake) \\ ${ }^{*}$ Corresponding author
}

To cite this article:

Yuichi Nodake, Ryoko Miura, Hirokazu Ryoya, Rina Momii, Satomi Toda, Ryuzo Sakakibara. Improvement of Lipid Metabolism and Ovalbumin-Induced Type I Allergy by Use of Soybean Milk Fermented by 16 Indigenous Lactic Acid Bacteria. Journal of Food and Nutrition Sciences. Vol. 4, No. 4, 2016, pp. 113-119. doi: 10.11648/j.jfns.20160404.17

Received: July 10, 2016; Accepted: July 18, 2016; Published: July 29, 2016

\begin{abstract}
A new fermented product of soybean milk (designated "PS-B1") was prepared by a four-step complex culture using 16 lactic acid bacteria of human intestinal origin. Because living lactic acid bacteria are completely removed by filtration of the fermented product, PS-B1 can be classified as a biogenic food. In the present study, we found that lipid accumulation was reduced significantly in 3T3-L1 adipocytes treated with PS-B1. Moreover, the production of IgE in response to ovalbumin was attenuated in rats fed with a diet containing PS-B1. Thus, PS-B1 represents one of the few biogenic food able to alter both lipid metabolism and ovalbumin-induced type I allergies, and is expected to find application in preventive medicine.
\end{abstract}

Keywords: Lactic Acid Bacteria, Soybean Milk, Biogenics, Lipid Metabolism, Ovalbumin-Induced Type I Allergy

\section{Introduction}

Probiotics are used around the world as foods for contributing to the enhancement of human health. In particular, probiotics fermented by lactic acid bacteria (LAB) have been used for centuries as traditional foods and medicines because of their multiple beneficial properties to human health and nutrition, including improvement of defecation, prevention of intestinal infection, and utilization of lactose [1-5]. Multiple reports indicate that oral administration of probiotics fermented by LAB provides moderate stimulation of the host's immune system and controls serum cholesterol levels [6-9]. Therefore, priority has been placed on obtaining new functional species and strains of LAB in order to develop new probiotics.

On the other hand, much attention has been paid to "biogenics" as a new concept in functional food. Biogenics are defined as bacterial metabolic products without living microorganisms and are thought to harbor special properties able to directly affect the living body via intestinal organs. For example, two tripeptides (valyl-prolyl-proline and isoleucyl-prolyl-proline) isolated from Lactobacillus helveticus-fermented milk have been shown to control hypertension by inhibiting angiotensin-converting enzyme [10]. Recent advances in the development of detection methods (such as metabolomic and nutrigenomic analyses) have facilitated the elucidation of the relationships between the ingestion of biogenics and health-enhancing effects.

Although many soybean milks fermented by LAB have been prepared and consumed around the world, we are aware of few reports showing that foods classified as biogenics are effective in improving both lipid metabolism and food allergy. In the present study, we prepared a new biogenic food, PS-B1, by fermenting soybean milk via a four-step complex culture using 16 indigenous LAB of human intestinal origin (Figure 1). LAB used in the preparation of PS-B1 subsequently was removed from the product by filtration. In order to evaluate the positive effects of PS-B1 on lipid metabolism and food allergy, we firstly examined whether PS-B1 reduces lipid accumulation in mouse 3T3-L1 adipocytes. We secondly tested the effect of administering a diet containing PS-B1 to rats immunized with ovalbumin (OVA), demonstrating that PS-B1 attenuated the induction of type I allergy (the amount of serum anti-OVA immunoglobulin ( $\mathrm{Ig}) \mathrm{E}$ ) in these animals. 


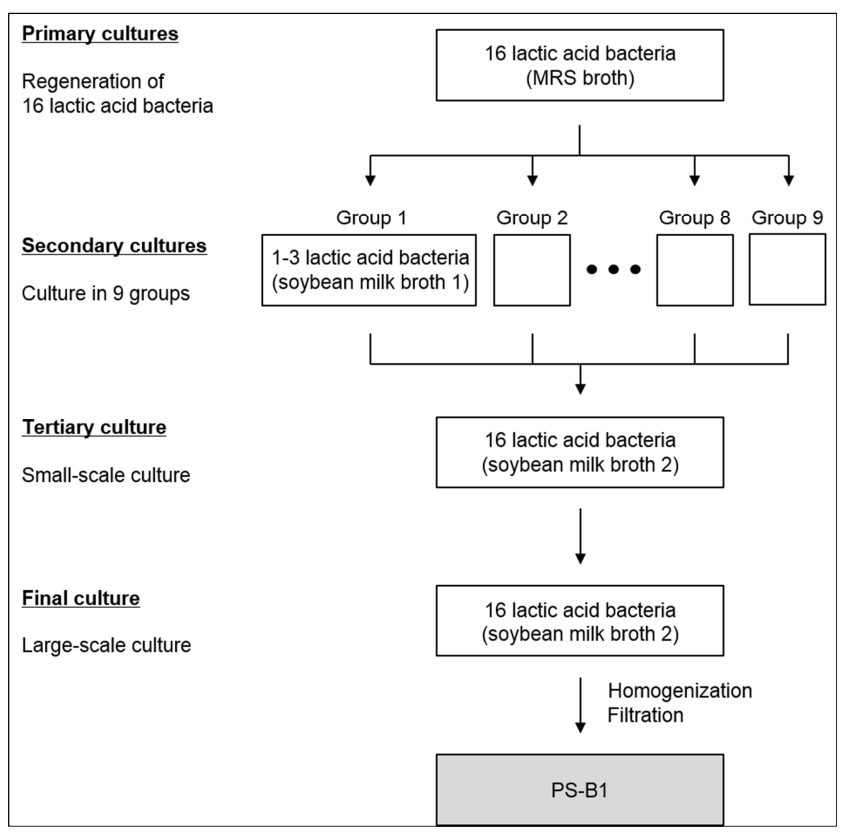

Figure 1. Preparation route of $P S-B 1 . P S-B 1$ was prepared as a fermented product of soybean milk by a four-step complex culture method using 16 indigenous lactic acid bacteria. Lactic acid bacteria used for preparation of PS-B1 and their distribution in the secondary cultures are shown in Table 1.

\section{Materials and Methods}

\subsection{Materials}

The mouse 3T3-L1 preadipocytes were purchased from DS Pharma Biomedical Co., Ltd. (Osaka, Japan). The rat IgE ELISA kit used for detection of IgE was purchased from Shibayagi Co., Ltd. (Shibukawa, Gunma, Japan). All other reagents used in this study were high-quality analytical-grade materials.

\subsection{Preparation of Soybean Milk Broths}

We obtained organically grown soybeans from Akama Farm (Obihiroshihoro, Hokkaido, Japan). After the soybeans were treated at $-2^{\circ} \mathrm{C}$ for 3 days and incubated at $10^{\circ} \mathrm{C}$ for $12 \mathrm{~h}$, a sterilized soybean milk was made from the soybeans in Nishikawaya Tofuten (Kawaguchi, Saitama, Japan).

In this study, two types of soybean milk broth were used for preparation of PS-B1. Soybean milk broth 1 (50\% soybean milk broth) was formulated in a mixture containing $6 \%$ skim milk, $0.1 \%$ glucose, and $0.1 \%$ yeast extract. Soybean milk broth 2 ( $80 \%$ soybean milk broth) was formulated in a mixture containing $2.4 \%$ skim milk, $0.5 \%$ glucose, and $0.4 \%$ yeast extract. Both broths were sterilized at $115^{\circ} \mathrm{C}$ for $15 \mathrm{~min}$ before use.

\subsection{Preparation of PS-B1}

PS-B1 was prepared from soybean milk broths by a four-step complex culture using 16 LAB, including organisms such as Lactobacilli, Bifidobacterium, and Enterococcus (Figure 1; Table 1). Individual LAB stored at $-80^{\circ} \mathrm{C}$ were aerobically pre-cultivated at $34^{\circ} \mathrm{C}$ in de Man-Rogosa-Sharpe (MRS) broth $(30 \mathrm{~mL} /$ bacteria) until the culture broth reached an optical density at $660 \mathrm{~nm}\left(\mathrm{OD}_{660}\right)$ of 1.3 ("primary cultures").

Table. 1. The 16 lactic acid bacteria used for preparation of PS-B1 and their grouping in secondary cultures.

\begin{tabular}{|c|c|c|c|}
\hline Groups & Lactic acid bacteria & & \\
\hline 1 & Lactobacillus brevis & $\begin{array}{l}\text { Lactobacillus } \\
\text { plantarum }\end{array}$ & $\begin{array}{l}\text { Enterococcus } \\
\text { faecium }\end{array}$ \\
\hline 2 & $\begin{array}{l}\text { Lactobacillus } \\
\text { delbrueckii }\end{array}$ & $\begin{array}{l}\text { Lactobacillus } \\
\text { helveticus }\end{array}$ & $\begin{array}{l}\text { Enterococcus } \\
\text { faecalis }\end{array}$ \\
\hline 3 & $\begin{array}{l}\text { Lactobacillus } \\
\text { gasseri }\end{array}$ & $\begin{array}{l}\text { Lactobacillus } \\
\text { rhamnosus }\end{array}$ & $\begin{array}{l}\text { Enterococcus } \\
\text { faecium }\end{array}$ \\
\hline 4 & $\begin{array}{l}\text { Lactobacillus } \\
\text { gasseri }\end{array}$ & $\begin{array}{l}\text { Lactobacillus } \\
\text { salivarius }\end{array}$ & $\begin{array}{l}\text { Enterococcus } \\
\text { durans }\end{array}$ \\
\hline 5 & $\begin{array}{l}\text { Lactococcus } \\
\text { garvieae }\end{array}$ & $\begin{array}{l}\text { Enterococcus } \\
\text { durans }\end{array}$ & $\begin{array}{l}\text { Enterococcus } \\
\text { faecium }\end{array}$ \\
\hline 6 & Lactobacillus casei & $\begin{array}{l}\text { Lactococcus lactis } \\
\text { subsp. }\end{array}$ & \\
\hline 7 & $\begin{array}{l}\text { Lactobacillus } \\
\text { delbrueckii subsp. }\end{array}$ & $\begin{array}{l}\text { Lactobacillus } \\
\text { paracasei subsp. }\end{array}$ & \\
\hline 8 & $\begin{array}{l}\text { Enterococcus } \\
\text { durans }\end{array}$ & $\begin{array}{l}\text { Enterococcus } \\
\text { faecium }\end{array}$ & \\
\hline 9 & $\begin{array}{l}\text { Lactobacillus } \\
\text { acidophilus }\end{array}$ & & \\
\hline
\end{tabular}

As summarized in Table 1, the $16 \mathrm{LAB}$ were divided into 9 groups consisting of 1-3 LAB each. Aliquots of MRS broth (1.5 mL/organism) from individual primary cultures were added to soybean milk broth $1(60 \mathrm{~mL})$ as shown in Table 1 . These soybean milk broths inoculated with bacteria of the 9 groups were incubated aerobically at $34^{\circ} \mathrm{C}$ for $24 \mathrm{~h}$ as small-scale cultures ("secondary cultures").

Next, aliquots of each of the 9 cultured broths (40 $\mathrm{mL}$ /group) obtained from secondary cultures were mixed in a sterile bottle. An aliquot $(10 \mathrm{~mL})$ of this mixture was added to $240 \mathrm{~mL}$ soybean milk broth 2 in a new sterile bottle, and the mixture was cultivated aerobically at $34^{\circ} \mathrm{C}$ for $24 \mathrm{~h}$ ("tertiary culture").

Finally, the entire volume of the tertiary culture was added into a sterile tray filled with fresh soybean milk broth $2(6.25 \mathrm{~L})$ and then cultured aerobically under the following sequential conditions: $34^{\circ} \mathrm{C}$ for $27 \mathrm{~h}, 40^{\circ} \mathrm{C}$ for $48 \mathrm{~h}$, and $65^{\circ} \mathrm{C}$ for $18 \mathrm{~h}$. The fermented product of this "final culture" was homogenized at $6,000 \mathrm{rpm}$ for $15 \mathrm{~min}$ using a homogenizer MEGATRON MT5000 (Central Scientific Commerce, Inc., Tokyo, Japan). The resulting suspension $(6.5 \mathrm{~kg})$ was transferred into a filtration-bag (Asahi Kasei Home Products Co., Ltd., Tokyo, Japan) and the filtrate was collected for 3 days. The resulting filtrate was filtered again using a $0.1-\mathrm{m}$ hollow fiber membrane (Jyujiya Co., Ltd., Okayama, Japan); the sterile product, designated "PS-B1", was lyophilized using a freeze dryer FDU-1200 (TOKYO RIKAKIKAI Co., Ltd., Tokyo, Japan). The lyophilized PS-B1 was employed in the following experiments.

\subsection{Cell Culture and Oil Red O Staining}

The mouse 3T3-L1 preadipocytes were seeded into 
$100-\mathrm{mm}$ tissue culture dishes at a density of $1.0 \times 10^{4}$ cells $/ \mathrm{cm}^{2}$ in Dulbecco's Modified Eagle's Medium (DMEM) supplemented with $10 \%$ calf serum; the plates then were incubated at $37^{\circ} \mathrm{C}$ and $5 \% \mathrm{CO}_{2}$ in a humidified incubator. Two days after reaching confluence (experimental day 0), the differentiation of 3T3-L1 preadipocytes into adipocytes was induced by the addition of $0.5 \mathrm{mM} \mathrm{3}$ - isobutyl - 1 - methyl xanthine, $0.25 \mu \mathrm{M}$ dexamethasone, $1 \mu \mathrm{g} / \mathrm{mL}$ insulin, and $10 \%$ fetal bovine serum (FBS). On day 2, the induction medium was replaced with DMEM containing $1 \mu \mathrm{g} / \mathrm{mL}$ insulin and $10 \%$ FBS. On day 4 , the medium was replaced with DMEM containing $10 \%$ FBS and PS-B1 $(0-750 \mu \mathrm{g} / \mathrm{mL})$; on day 6 , the spent medium was replaced with fresh DMEM + FBS containing the same respective concentration of PS-B1.

On day 8 , the cells were washed twice with phosphate-buffered saline at $\mathrm{pH} 7.4$ (PBS), fixed by incubation with $10 \%$ formaldehyde in PBS for $10 \mathrm{~min}$ at room temperature, and then stained with $4 \mathrm{~mL} /$ plate of oil red $\mathrm{O}$ solution $(1.8 \mathrm{mg} / \mathrm{mL}$ oil red $\mathrm{O}$ reagent in $60 \%$ isopropanol) for $20 \mathrm{~min}$ at room temperature. Excess stain was removed by washing twice with distilled water and oil red $\mathrm{O}$ internalized in lipid droplets was dissolved by the addition of $7 \mathrm{~mL} /$ plate of isopropanol. The absorbance of the dissolved dye was measured at $510 \mathrm{~nm}$ using a Tecan infinite M200 plate reader.

\subsection{Ingestion of PS-B1}

An animal experiment was conducted in accordance with the "Standards Relating to the Care and Management of Laboratory Animals and Relief of Pain" (Notice No. 88, Ministry of the Environment, Government of Japan). The experimental protocol in the animal experiments was approved by the Ethics Review Committee of Nagasaki International University (Approval No. 25). The schedule of the animal experiment is shown in Figure 2. Male Kud: Wistar rats at 4 weeks of age (Kyudo Co., Kumamoto, Japan) were individually housed in a room with controlled temperature, relative humidity, and light $\left(23 \pm 2^{\circ} \mathrm{C}, 60 \pm 2 \%\right.$ $\mathrm{RH}$, and 7:00 to 19:00 $\mathrm{h}$, respectively). The rats had free access to water and the CE-2 powder diet (CLEA Japan, Inc., Tokyo, Japan). After a week of acclimatization, the rats were randomly divided into four groups of five rats each. The control groups of rats were fed ad libitum with CE-2 powder diet (Groups I and II). For the experimental groups, the CE-2 powder diet was replaced (at 14 or 0 days after the acclimatization; Group III and IV, respectively) with a basal powder diet supplemented with 3\% lyophilized PS-B1. General signs were observed daily, and the body weight of each rat was measured once a week. The amount of feed ingestion was estimated by weighing the leftovers each week.

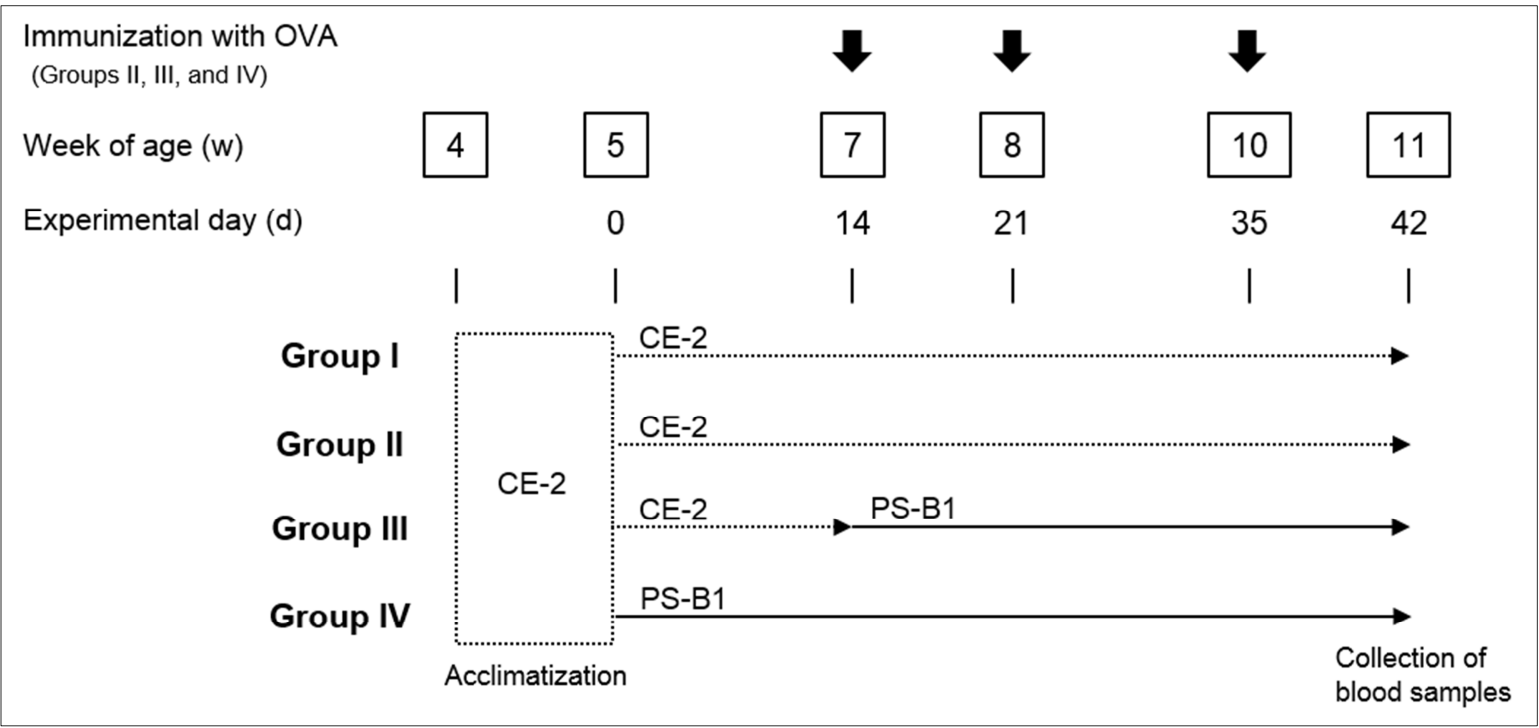

Figure 2. Schedule of ingestion of PS-B1 and immunization with OVA in animal experiment. Male Kud: Wistar rats at 4 weeks of age were randomly divided into four groups ( $n=5$ /group) after a week of acclimatization. The rats in Groups I and II were fed with CE-2 powder diet. In Groups III and IV, CE-2 supplemented with 3\% lyophilized PS-B1 was given to rats from 14 or 0 days after the acclimatization, respectively. In rats of Groups II, III, and IV, immunization with OVA was performed 14,21, and 35 days after the acclimatization; rats of Group I were immunized with vehicle on the same schedule. Blood samples were collected from all rats at one week after the final immunization.

\subsection{Immunization with $O V A$}

Immunization of rats was performed by using OVA as a soluble antigen. Briefly, a mixture of $1 \mathrm{mg} / \mathrm{mL}$ of OVA in PBS $(100 \mu \mathrm{L})$ and Imject Alum (Pierce Biotech., Inc., Rockford, Illinois, USA) was injected intraperitoneally into rats in Groups II, III, and IV at two weeks after acclimatization (Figure 2). Booster injections of the same amount of OVA in Imject Alum were administered 7 and 21 days after the initial injection. In Group I, animals were immunized on the same schedule but with equivalent volumes of a mixture of PBS (containing no antigen) and alum. Blood samples were collected from the immunized rats one week after the final injection. Following clotting and centrifugation, the resulting supernatant (antiserum) was stored at $-20^{\circ} \mathrm{C}$ until used in the enzyme-linked immunosorbent assay (ELISA). 


\subsection{Detection of Anti-OVA IgE}

To measure the amount of anti-OVA IgE in serum, the detection assay was performed using the rat IgE ELISA kit according to the instruction manual. The absorbance of the resulting reaction mixture in a 96-well microtiter plate was read at $414 \mathrm{~nm}$ on a Tecan infinite M200 plate reader.

\subsection{Statistical Analysis}

Each assay in detection of accumulated lipid and anti-OVA $\mathrm{IgE}$ was performed in triplicate; resulting data are presented as mean \pm standard error. Statistical significance was evaluated using a two-tailed Student's t-test; $p$ values of < 0.05 were considered statistically significant.

\section{Results and Discussion}

\subsection{Preparation of PS-B1}

In this study, PS-B1 was prepared as a biogenic food from soybean milk by a four-step complex culture using $16 \mathrm{LAB}$ (Figure 1). Primary cultures were performed using MRS broth for regenerating stored bacteria. In the secondary cultures, 9 individual groups of LAB were grown in separate aliquots of soybean milk broth 1 (Table 1). In the tertiary culture, aliquots of each of the secondary cultures were combined and then used as an inoculum for a bottle of soybean milk broth 2; the resulting mixture was grown as a small-scale culture. For the final culture, the tertiary culture was used to inoculate a large volume of soybean milk broth 2 , yielding a large-scale culture. The final culture was subjected to filtration through a $0.3-\mu \mathrm{m}$ filter, yielding a sterile product designated PS-B1. PS-B1 was expected to include not only soybean milk and components of killed microorganisms but also metabolites secreted by LAB. Multiple reports have indicated that the substances obtained from LAB fermentation provide health benefits [11-13]; we therefore assessed the health potential of the comprehensive mixture of substances included in PS-B1. Specifically, we used this novel fermented soybean milk product as biogenics in the following experiments.

\subsection{Suppression Effect of PS-B1 on Lipid Accumulation}

We evaluated the influence of PS-B1 on lipid accumulation in 3T3-L1 adipocytes, as assessed by oil red $\mathrm{O}$ staining. The differentiation of 3T3-L1 preadipocytes into adipocytes was induced by treatment with a mixture of 3-iso-butyl-1-methylxanthine, dexamethasone, and insulin; induced cells then were cultured for 4 days in DMEM medium containing PS-B1 at various concentrations. As shown in Figure 3, cells grown in medium containing PS-B1 accumulated significantly less oil red $\mathrm{O}$ compared to those grown in medium lacking the supplement; the effect of PS-B1 was nominally dose dependent. Specifically, when 3T3-L1 adipocytes were treated with PS-B1 at a concentration of 500 $\mu \mathrm{g} / \mathrm{mL}$, lipid accumulation was suppressed by approximately $16 \%$ compared to the control. Thus, supplementation of growth medium with PS-B1 yielded decreased lipid accumulation during in vitro growth of 3T3-L1 adipocytes.

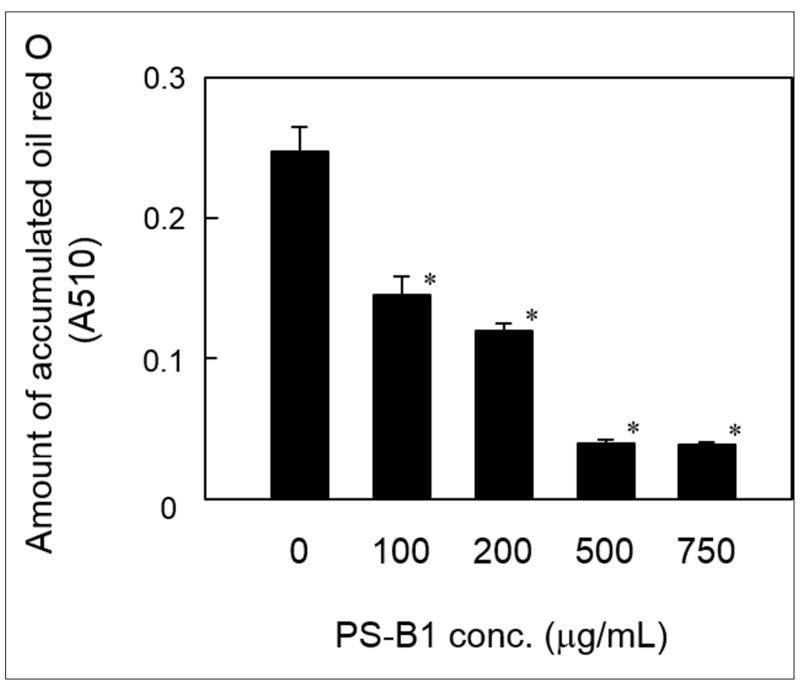

Figure 3. Influence of PS-B1 on lipid accumulation in 3T3-L1 adipocytes. 3T3-L1 adipocytes were treated with PS-B1 at the indicated concentrations for 4 days. The lipid accumulated in 3T3-L1 adipocytes was detected by oil red $O$ staining. The stained oil red $O$ was extracted with isopropanol. The absorbance of the extracted oil red $O$ was spectrophotometrically determined at $510 \mathrm{~nm}$ to measure lipid accumulation. Experimental data are presented as mean \pm standard error, and significant differences (using two-tailed Student's t-tests) from the values in the absence of PS-B1 are indicated by *p $<0.05$.

In general, it is important to control lipid accumulation in adipocytes in order to maintain the levels of adipocytokines, such as adiponectin and leptin. Because food and food components contributing to the control of lipid accumulation are expected to be employed as anti-obesity foods, the elucidation of physiological mechanisms in their bioactivities are required. For example, Yi-Gan-San, which is a Chinese herbal medicine, has been shown to suppress lipid synthesis and fat accumulation in adipocytes through modulation of the activities of two transcription factors, sterol regulatory element-binding protein-1c (SREBP-1c) and forkhead box class O1 (FoxO1) [14]. The former transcription factor is the main regulator of the synthesis of fatty acids and cholesterol, and the latter is involved in adipocyte differentiation. In the present study, our results suggested that PS-B1 may include substances that suppress lipid synthesis or accelerate lipid degradation. In future work, we plan to investigate the molecular mechanism of action of PS-B1.

\subsection{Influence of PS-B1 on Growth of Rats}

We conducted an animal experiment examining the possibility of PS-B1 as biogenic therapy for the treatment of type I allergies, here modeled as the induction of an $\operatorname{IgE}$ response following immunization with OVA (Figure 2). In this animal experiment, the rats of Groups I and II were control animals, provided with $a d$ lib access to the CE-2 powder diet lacking PS-B1. Rats of the latter group were immunized with OVA. The rats in Groups III and IV were provided with ad lib access to the CE-2 powder diet supplemented with $3 \%$ lyophilized PS-B1 for 4 and 6 weeks, respectively. The rats in 
each of the groups exhibited similar changes in body weight; all animals grew well without marked differences in appearance (data not shown). No unscheduled mortality occurred in any of the groups during the study interval. These results indicated that the ingestion of PS-B1 at concentrations of up to $3 \%$ does not influence the growth of rats, confirming the safety of PS-B1. We note, however, that this result could be considered to contrast with the above observation (in Figure 3 ) that in vitro exposure of mouse adipocytes to PS-B1 resulted in significant decreases in lipid accumulation. PS-B1 may contribute to the normalization of lipid metabolism in obesity. Therefore, it will be critical to conduct further animal experiments testing PS-B1 supplementation in animals genetically predisposed to obesity and/or in animals maintained on high-fat or high-calorie diets. We hypothesize that PS-B1 effects on weight gain will be more readily detected in animal models of obesity.

\subsection{Suppression Effect of PS-B1 on Production of Anti-OVA IgE}

The amount of IgE response to OVA in serum obtained from rats in each group is shown in Figure 4. Comparison between Groups I and II showed that immunization with OVA yielded an approximately 9.8-fold increase in IgE levels, indicating that immunization was sufficient to stimulate $\operatorname{IgE}$ production in this model. Ingestion of PS-B1 yielded significant attenuation of IgE levels (Groups III and IV compared to Group II). Moreover, the amount of $\operatorname{IgE}$ in Group IV was significantly lower than that in Group III. Notably, PS-B1 was provided to rats in Group IV before immunization with OVA, while immunization and ingestion of PS-B1 were initiated on the same day for Group III. These results suggested that pre-ingestion of PS-B1 provides more effective attenuation of the activation of IgE production.

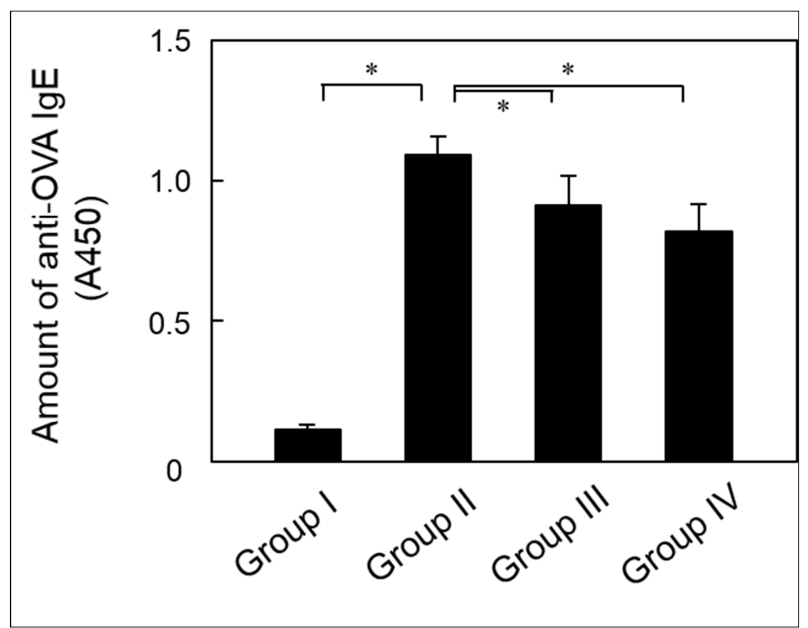

Figure 4. Influence of PS-B1 on anti-OVA IgE production. In the animal experiment, blood samples were collected from all rats at one week after the final immunization. The amounts of anti-OVA $\operatorname{IgE}$ in serum prepared from rats of the four groups were detected by ELISA. The absorbances of the resulting reaction mixtures at $414 \mathrm{~nm}$ were measured on the plate reader. Experimental data are presented as mean \pm standard error, and significant differences (using two-tailed Student's t-tests) from the values in Group I or II are indicated by $* p<0.05$.
Most allergic diseases associated with immediate hypersensitivity are closely related to acceleration of $\operatorname{IgE}$ production. In general, the production of IgE results in type I allergy, owing to dominance of Th2-type immune responses. It is also known that the enhancement of Th1-type immunity suppresses Th2-type immune responses. In the present study, we investigated only the effect of PS-B1 on OVA-induced type I allergy. Further analysis will be needed to address the possible influence of PS-B1 on cytokines such as interferon (IFN)- $\gamma$, interleukin (IL)-4, IL-10, and IL-12; these factors may be involved in PS-B1's effect on the production of anti-OVA IgE. For example, there are reports that the daily ingestion of Lactobacillus plantarum strain L-137 or KW3110 suppresses IgE production in vivo through IL-12 production, which induces the Th1-type immune response [15, 16]. In another example, a carbohydrate was shown to reduce the serum IgE level by suppression of IL-4 production and inactivation of Th2 response via the intestinal immune system [17]. Based on our results, we hypothesize that PS-B1 may increase Th1 cytokines and decrease Th2 cytokines.

PS-B1 is expected to include not only components of soybean milk and LAB-secreted metabolites, but also various LAB cell debris, including cell wall components (e.g., lipoteichoic acid and peptidoglycan) and genetic material (e.g., unmethylated $\mathrm{CpG}$ motifs from LAB DNA) [18-21]. Therefore, it is possible that the effects of PS-B1 are mediated by the direct influence of these substances on immune cells. On the other hand, oral L-92 administration has been reported to regulate both $\mathrm{Th} 1$ and $\mathrm{Th} 2$ cytokine responses, to suppress serum anti-OVA IgE, and to induce transforming growth factor (TGF)- $\beta$ production in Peyer's patch cells [22]. In vivo, PS-B1 may activate the intestinal immune system represented by Peyer's patch cells and/or alter intestinal microbiota.

We have additional data supporting the effect of PS-B1 on the improvement of OVA-induced type I allergy. In a metabolomic analysis (currently in progress) of LAB-metabolized soybean milk, we have observed elevated levels of cepharanthine (data not shown), an alkaloid known to indicate anti-inflammatory, anti-oxidative, anti-allergic, and immunomodulatory activities [23, 24]. Macrophages have been shown to produce $\mathrm{Th} 2$ cytokines when in an oxidative environment and to produce Th1 cytokines when in a reductive environment $[25,26]$; thus the presence of antioxidants in PS-B1 may contribute to mucosal immunity.

\section{Conclusion}

In this study, we prepared PS-B1, a biogenic fermented product of soybean milk that is generated by a four-step complex culture using 16 indigenous LAB. Subsequent analysis demonstrated that PS-B1 improves lipid metabolism in vitro and attenuates OVA-induced type I allergy in vivo. The precise molecular mechanism (s) responsible for the effects of PS-B1 on the lipid accumulation and the $\operatorname{IgE}$ production remain undefined. Since we expect that PS-B1 is a candidate for daily use as a biogenic food suitable for preventive medicine, further investigations will be needed to 
address these and further issues.

\section{Acknowledgments}

The authors express their sincere thanks to Dr. Fukasawa (Nagasaki International University, Sasebo, Nagasaki, Japan) for providing experimental information on ELISA. This work was supported by a grant-in-aid for scientific research from Ministry of Education, Culture, Sports, Science, and Technology of Japan (Grant No. 24700854).

\section{References}

[1] Rao D. R., Chawan C. B., and Pulusani S. R., Influence of milk and thermophilus milk on plasma cholesterol levels and hepatic cholesterogenesis in rats. J. Food Sci., 46, 1339-1341 (1981).

[2] Wright R. S., Anderson J. W., and Bridge S. R., Propionate inhibits hepatocyte lipid synthesis. Proc. Soc. Exp. Biol. Med., 195, 26-29 (1994).

[3] Agerbaek M., Gerdes L. U., and Richelsen B., Hypocholesterolaemic effect of a new fermented milk product in healthy middle-aged men. Eur. J. Clin. Nutr., 49, 346-352 (1995).

[4] Cano P. G., Agüero G., and Perdigón G., Immunological effects of yogurt addition to a re-nutrition diet in a malnutrition experimental model. J. Dairy Res., 69, 303-316 (2002).

[5] "Yogurt: nutritional and health properties," ed. by Chandan R. C., National Yogurt Association, McLean, Virginija (1989).

[6] Perdigón G., Nader de Macías M. E., Alvarez S., Pesce de Ruiz Holgado A., and Oliver G., Effect of perorally administered lactobacilli on macrophage activation in mice. Infect. Immun., 53, 404-410 (1986).

[7] De Simone C., Vesely R., Negri R., Bianchi Salvadori B., Zanzoglu S., Cilli A., and Lucci L., Enhancement of immune response of murine Peyer's patches by a diet supplemented with yogurt. Immunopharmacol. Immunotoxicol., 9, 87-100 (1987).

[8] Perdigón G., Nader de Macías M. E., Alvarez S., Oliver G., and Pesce de Ruiz Holgado A., Prevention of gastrointestinal infection using immunobiological methods with milk fermented with L. casei and L. acidophilus. J. Dairy Res., 57, 255-264 (1990).

[9] Bosch M., Fuentes M. C., Audivert S., Bonachera M. A., Peiró S., Cuñé J., Lactobacillus plantarum CECT 7527, 7528 and 7529: probiotic candidates to reduce cholesterol levels. J. Sci. Food Agric., 94, 803-809 (2014).

[10] Nakamura Y. and Ohki K., Effect of tripeptides produced by Lactobacilli on blood pressure and vascular endothelial function. J. Intest. Microbiol., 24, 259-264 (2010).

[11] Ménard S., Candalh C., Bambou J. C., Terpend K., Cerf-Bensussan N., Heyman M., Lactic acid bacteria secrete metabolites retaining anti-inflammatory properties after intestinal transport, Gut, 53, 821-828 (2004).
[12] Zeuthen L. H., Christensen H. R., Frøkiær H., Lactic acid bacteria inducing a weak interleukin-12 and tumor necrosis factor alpha response in human dendritic cells inhibit strongly stimulating lactic acid bacteria but act synergistically with gram-negative bacteria. Clin. Vaccine Immunol., 13, 365-375 (2006).

[13] Heuvelin E., Lebreton C., Grangette C., Pot B., Cerf-Bensussan N., and Heyman M., Mechanisms involved in alleviation of intestinal inflammation by Bifidobacterium breve soluble factors. PLoS ONE, 4, e5184 (2009).

[14] Izumi M., Seki T., Iwasaki K., Sakamoto K., Chinese herbal medicine Yi-Gan-San decreases the lipid accumulation in mouse 3T3-L1 adipocytes by modulating the activities of transcription factors SREBP-1c and FoxO1. Tohoku J. Exp. Med., 219, 53-62 (2009).

[15] Hirose Y., Murosaki S., Yamamoto Y., Yoshikai Y., and Tsuru T., Daily intake of heat-killed Lactobacillus plantarum L-137 augments acquired immunity in healthy adults. J. Nutr., 136, 3069-3073 (2006).

[16] Ichikawa S., Miyake M., Fujii R., and Konishi Y., Orally administered Lactobacillus paracasei KW3110 induces in vivo IL-12 production. Biosci. Biotechnol. Biochem., 73, 15611565 (2009).

[17] Nagura T., Hachimura S., Hashiguchi M., Ueda Y., Kanno T., Kikuchi H., Sayama K., Kaminogawa S., Br. J. Nutr., 88, 421426 (2002).

[18] Peña J. A. and Versalovic J., Lactobacillus rhamnosus GG decreases TNF-alpha production in lipopolysaccharide-activated murine macrophages by a contact-independent mechanism, Cell Microbiol., 5, 277-285 (2003).

[19] Matsuguchi T., Takagi A., Matsuzaki T., Nagaoka M., Ishikawa K., Yokokura T., and Yoshikai Y., Lipoteichoic acids from Lactobacillus strains elicit strong tumor necrosis factor alpha-inducing activities in macrophages through Toll-like receptor 2. Clin. Diagn. Lab. Immunol., 10, 259-266 (2003).

[20] Sparwasser T., Koch E. S., Vabulas R. M., Heeg K., Lipford G. B., Ellwart J. W., and Wagner H., Bacterial DNA and immunostimulatory $\mathrm{CpG}$ oligonucleotides trigger maturation and activation of murine dendritic cells. Eur. J. Immunol., 28, 2045-2054 (1998).

[21] Iliev I. D., Kitazawa H., Shimosato T., Katoh S., Morita H., He F., Hosoda M., and Saito T., Strong immunostimulation in murine immune cells by Lactobacillus rhamnosus GG DNA containing novel oligodeoxynucleotide pattern. Cell Microbiol., 7, 403-414 (2005).

[22] Torii A., Torii S., Fujiwara S., Tanaka H., Inagaki N., and Nagai H., Lactobacillus acidophilus strain L-92 regulates the production of Th1 cytokine as well as Th2 cytokines. Allergol. Int., 56, 293-301 (2007).

[23] Furusawa S. and Wu J., The effects of biscoclaurine alkaloid cepharanthine on mammalian cells: implications for cancer, shock, and inflammatory diseases. Life Sci., 80, 1073-1079, (2007).

[24] Rogosnitzky M. and Danks R., Therapeutic potential of the biscoclaurine alkaloid, cepharanthine, for a range of clinical conditions. Pharmacol. Reports, 63, 337-347 (2011). 
[25] Park B. G., Jung H. J., Cho Y. W., Lim H. W., Lim C. J., Potentiation of antioxidative and anti-inflammatory properties of cultured wild ginseng root extract through probiotic fermentation. J. Pharm. Pharmacol., 65, 457-464, (2013).
[26] Murata Y., Shimamura T., and Hamuro J., The polarization of T (h) $1 / \mathrm{T}$ (h) 2 balance is dependent on the intracellular thiol redox status ofmacrophages due to the distinctive cytokine production, Int. Immunol., 14, 201-212 (2002). 